\title{
Non-suicidal self-injury among sexual minority youth: An etiological and treatment overview
}

\author{
Lauren C. Smithee
}

Virginia Tech

Brock W. Sumner

Brigham Young University - Provo

Roy A. Bean

Brigham Young University - Provo, roy_bean@byu.edu

Follow this and additional works at: https://scholarsarchive.byu.edu/facpub

Part of the Other Social and Behavioral Sciences Commons

\section{Original Publication Citation}

*Smithee, L. C., *Sumner, B. W., \& Bean, R. A. (2018). Non-suicidal self-injury among sexual minority youth: An etiological and treatment overview. Children and Youth Services Review, 96, 212-219.

\section{BYU ScholarsArchive Citation}

Smithee, Lauren C.; Sumner, Brock W.; and Bean, Roy A., "Non-suicidal self-injury among sexual minority youth: An etiological and treatment overview" (2018). Faculty Publications. 5033.

https://scholarsarchive.byu.edu/facpub/5033 


\title{
Non-suicidal self-injury among sexual minority youth: An etiological and treatment overview
}

\author{
Lauren C. Smithee ${ }^{\mathrm{a}, *}$, Brock W. Sumner ${ }^{\mathrm{b}}$, Roy A. Bean ${ }^{\mathrm{c}}$ \\ ${ }^{\text {a }}$ Marriage and Family Therapy, Virginia Tech, United States \\ ${ }^{\mathrm{b}}$ Marriage and Family Therapy, Brigham Young University, United States \\ ${ }^{\mathrm{c}}$ Marriage and Family Therapy, LMFT, Brigham Young University, United States
}

\section{A B S T R A C T}

Non-suicidal self-injury (NSSI) among sexual minority youth (SMY) is a problem that is growing in attention yet is understudied and requires an informed response from therapists who engage in clinical work with youth. This paper proposes a unique model of treatment for working with SMY who engage in NSSI, accounting for proximal, distal, and systemic factors that influence the etiology of NSSI. This model is based on a review of two bodies of clinical literature: (a) treating adolescent NSSI and (b) working with SMY and their families. As an etiological theory of self-harm, Nock (2009) is applied to SMY, factoring in the unique stressors experienced by this group that affect intervention. Reviewed treatment approaches include Dialectical Behavioral Therapy for Adolescents (DBT-A) and narrative family therapy. Treatment guidelines are conceptualized and outlined to aid therapists in providing affirmative and informed therapeutic services. While few, if any articles have studied treatment for SMY who engage in NSSI, this article contributes to the literature by proposing culturally competent interventions.

\section{Introduction}

As a growing phenomenon among adolescents, non-suicidal selfinjury (NSSI), a type of physical injury to the body without a deliberate suicide attempt, is associated with major costs to youth, families and health care providers (Sinclair, Gray, Rivero-Arias, Saunders, \& Hawton, 2011; Umbach, 2013). Accumulating empirical evidence demonstrates that NSSI behaviors are either increasing in prevalence among adolescents, or are gaining more attention within research, and this is disproportionately true among sexual minority youth (SMY) (Fox et al., 2017). Over the last several decades, as visibility of NSSI behaviors has increased, there has been the accompanying development of etiological theory and an evolution of effective treatment modalities. Nevertheless, it is important to note that very little of the NSSI related research findings have been applied to specific subpopulations. As such, the purpose of this article is to examine the relevant literature on NSSI and apply it directly to sexual minority youth, a population that includes adolescents with lesbian, gay, bisexual, pansexual, queer, and other non-heterosexual identities.

It is important to note that "LGBTQ +" has served as an umbrella term for gender and sexual minority populations. While transgender and gender non-conforming (TGNC) youth often share similar experiences with SMY (especially if a youth identifies as both a gender and sexual minority), there are also unique differences in the experiences of these populations. While other social identities such as racial, ethnic, and gender identity are certainly important to consider when conceptualizing the experiences of youth experiencing minority stress, this review will focus solely on stigmatized sexuality as opposed to gender identity. Therapists who desire to apply the information within this article to their clinical work with TGNC youth are advised to consult the current WPATH Standards of Care (World Professional Association for Transgender Health, 2018) for effective and ethical clinical practice. Before applying the principles within this review to TGNC youth, clinicians are admonished to further educate themselves on (a) differences between gender identity, sexual orientation, and sex and (b) the unique experiences TGNC youth have with gender dysphoria, internalized transphobia, and potential transitioning.

\section{Method}

The current article is a literature review of two distinct bodies of clinical literature: treatment of adolescent NSSI and working with SMY youth and their families. The search terms used to identify the articles for review included but were not limited to "adolescent treatment for self-injury", "family therapy for self-injury", "self-harm assessment", "sexual minority stress", "LGBT minority stress," "LGBT youth," and "therapy for LGBT youth." These search terms enabled the researchers to develop a holistic picture of these two distinct bodies of literature and draw important intervention recommendations. The structure of the review focuses first on the theories underlying NSSI (origins and

\footnotetext{
*Corresponding author at: Virginia Tech, 260 Wallace Hall, 295 West Campus Drive, Blacksburg, VA 24061, United States.

E-mail address: Lsmithee@vt.edu (L.C. Smithee).
} 
maintenance) and noting the unique factors that impact SMY. Next, specific assessments and treatment recommendations are detailed and incorporated into their respective theoretical frameworks. This review will allow clinicians to gain understanding of the theoretical implications of working with NSSI and SMY populations as well as theoretically-based treatment of NSSI within SMY populations.

\section{NSSI research and theory}

Research indicates that the measurement and reporting of NSSI behaviors in youth has increased dramatically over the last fifty years with prevalence rates estimated as low as $1 \%$ just 45 years ago (Whitehead, Johnson, \& Ferrence, 1973). By comparison, recent estimates of adolescent NSSI range from $15 \%$ to $20 \%$ (Klonsky, Victor, \& Saffer, 2014; Muehlenkamp, Brausch, Quigley, \& Whitlock, 2012; Zetterqvist, 2017). Additional support for its increased prevalence is found in the fact that less than $6 \%$ of adults report a history of NSSI (Klonsky et al., 2014) whereas, the percentage of NSSI in adolescents today is three-to-five times more (15-to-20\%; Klonsky et al., 2014; Zetterqvist, 2017). Regardless of whether this discrepancy could be attributed to an under-reporting bias in the adult population, increased visibility in research on NSSI, or a reflection of changing trends in adolescent behavior, this phenomenon warrants additional attention (Nock, 2009).

Research has established that the onset of NSSI behaviors is most typical during early adolescence (ages of 13-15), making it particularly important to study for early-to-middle adolescents (Klonsky et al., 2014). Another critical group distinction is that while the onset and presence of NSSI behaviors appear to occur independent of sex, ethnicity, and socioeconomic status (Glenn \& Klonsky, 2009), youth who identify as a sexual minority are at a higher risk for NSSI compared to heterosexual peers, due to sexual-orientation specific stressors (Fox et al., 2017). Furthermore, NSSI behaviors are more severe and occur more frequently among those who identify as a sexual minority (Fox et al., 2017). The overall increased prevalence of NSSI behaviors and its disproportionate prevalence within the SMY population highlights the need for effective treatment and accurate understanding of the issue by clinicians.

While NSSI is, in and of itself, a serious mental and physical health issue, its connection to suicide risk (a significant cross-sectional and longitudinal predictor) further accentuates its importance (Hamza, Stewart, \& Willoughby, 2012; Whitlock \& Knox, 2007). Recent literature states that NSSI is a robust risk factor for suicide across the lifespan (Scott, Pilkonis, Hipwell, Keenan, \& Stepp, 2015) as well as posing a serious concern for unintentional harm or accidental fatality (Lewis \& Heath 2015; Whitlock \& Knox, 2007). NSSI is associated with pronounced risks that underscore the importance of understanding the behaviors origins and its maintenance.

\section{Etiological theory associated with NSSI}

Fundamentally, NSSI is a counterintuitive behavior, as it is difficult to explain why an individual would desire to cause themselves pain. Accompanying this confusion and perhaps contributing to it, are several competing hypotheses set on explaining NSSI behaviors, including "social learning hypothesis," self-punishment hypothesis," "social signaling hypothesis," and "pragmatic hypothesis" (Muehlenkamp et al., 2012; Nock, 2009; Nock, 2010). Correcting for the lack of consensus, Nock (2009) and Muehlenkamp et al. (2012) have proposed more comprehensive etiological theories, suggesting that NSSI serves an emotional regulatory function while also indirectly communicating emotional distress to others interpersonally (see Fig. 1). These theoretical frameworks integrate the existing literature and provide clear direction for the intervention recommendations that will be presented below. Furthermore, Nock's (2009) theory has been tested and empirically validated repeatedly (Hooley \& Franklin, 2018); because of its ease of application and widespread validation Nock's theory will be the foundation of the recommended interventions for treatment. While the function of NSSI may vary from person to person, it is important to become familiar with the two main types of predictive effects that contribute to the selection of NSSI as a coping mechanism: those effects which trigger a NSSI episode (proximal) and those effects which predispose individuals to the selection of NSSI behaviors as a general strategy of coping (distal).

The proximal effects are the stressors which immediately precede the NSSI behavior and "cause" the intrapersonal distress and emotional dysregulation that must be managed through NSSI (Meyer, Teylan, \& Schwartz, 2015; Muehlenkamp et al., 2012; Nock, 2009; Nock, 2010). Proximal effects are only limited by perception, as anything that creates intrapersonal distress has the potential to be a proximal effect for an individual. Nevertheless, some of the most frequent proximal effects are depression, anxiety, a lack of social support, social isolation, substance abuse, troubled relationships, poor school performance, and low selfesteem (Meyer et al., 2015; Muehlenkamp et al., 2012). These factors may appear interrelated (e.g. depression influencing further social isolation), however, whatever the primary cause of intrapersonal distress is that precedes NSSI is considered a proximal effect.

While proximal effects are hypothesized to lead to emotional dysregulation, which may trigger NSSI behaviors, distal effects are those events which predispose an individual to select NSSI as a specific strategy for emotional regulation (Nock, 2009). These effects typically occur (or develop) early in the individual's life (e.g., childhood abuse, predisposition to emotional reactivity, introversion, and negative thought patterns; Nock, 2009; Prinstein et al., 2010). These factors may contribute to problems with affect regulation and create disturbances in interpersonal communication, increasing the likelihood of resorting to NSSI as a coping strategy to emotionally regulate and indirectly communicate distress to others (Muehlenkamp et al., 2012; Nock, 2009; Prinstein et al., 2010). Furthermore, distal factors can prevent the development of effective social, problem solving, and communication skills (Prinstein et al., 2010).

After the first NSSI incident there is an increased likelihood of subsequent NSSI partially due to NSSI having utility in terms of emotional regulation and interpersonal communication (Muehlenkamp et al., 2012; Nock, 2009). Additionally, as seen in Fig. 1, there is often a cyclical nature associated with NSSI; where each act may provide momentary emotional relief but also elicit intrapersonal distress in the form of guilt, regret, and/or shame (Houben et al., 2017). The resultant distress following a NSSI episode may then function as a proximal effect, perpetuating the use of NSSI for emotional regulation (Houben et al., 2017; Meyer et al., 2015; Nock, 2009). Consequently, while some youth only temporarily experiment with NSSI and do not continue the behavior, for many individuals, NSSI is not an isolated incident but rather a series of recurring behaviors.

\section{NSSI and sexual minority youth (SMY)}

\subsection{Discrimination, oppression, and minority stress}

\subsubsection{Theoretical frameworks}

Several theories exist on the etiology of disparities in health and stress among SMY, including minority stress theory (Meyer, 2003). Meyer's describes minority stress as excess stress stigmatized populations experience due to their identities (Meyer, 2003). Minority stress is conceptualized as a mediator between stigmatized identity status and negative health outcomes, including stress related to non-adherence to heteronormative standards (Meyer, 2003; Pitoňák, 2017). Another theory that has been used to address minority stress is syndemic theory, which posits that as sexual minorities experience marginalization, they tend to develop psychological difficulties, such as depression and poor self-esteem (Pachankis, 2015; Stall, Friedman, \& Catania, 2007). These psychosocial health disparities often co-occur and cause an additive 


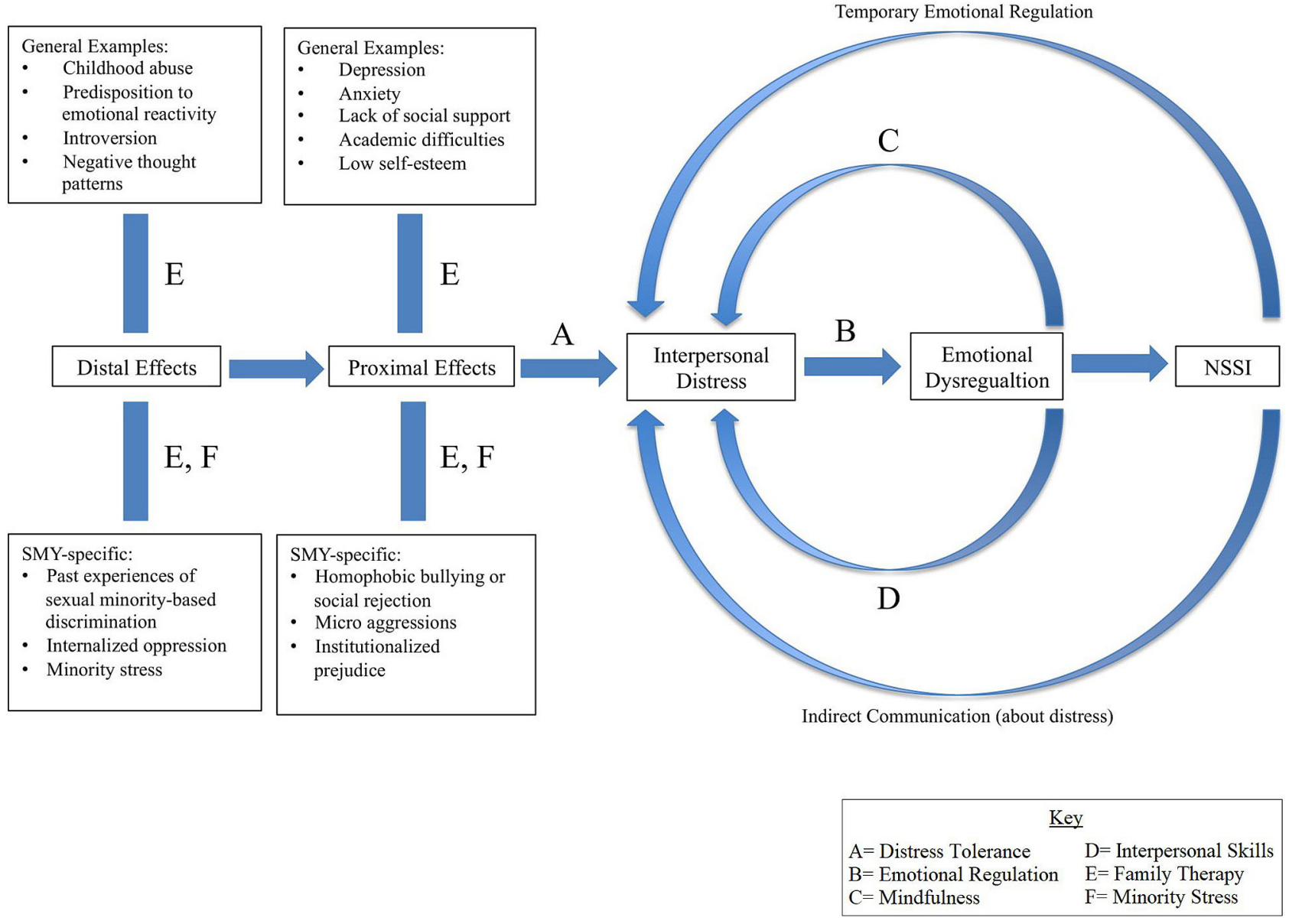

Fig. 1. Suggested etiological and treatment overview of non-suicidal self-injury (NSSI) among sexual minority youth (SMY).

effect, increasing a SMY's risk for negative health outcomes (Pachankis, 2015; Stall, Friedman, \& Catania, 2007). In contrast, Hatzenbuehler's psychological mediation framework (2009) describes how sexual minorities experience increased stress resulting from stigma, which elevates risk for emotional dysregulation and interpersonal difficulties. In turn, these processes mediate the relationship between minoritystress-related psychopathology (Hatzenbuehler, 2009). While these main frameworks differ in how they theorize about the etiology of health disparities among SMY, they all provide insight into how stigma and psychological health disparities are related, compounding the likelihood for NSSI.

\subsubsection{SMY, stigma, and psychological health}

Research consistently finds that SMY experience disproportionate stressors, including higher rates of harassment, discrimination, and social rejection compared to heterosexuals (DeCamp \& Bakken, 2016; Meyer et al., 2015; Muehlenkamp, Hilt, Ehlinger, \& McMillan, 2015; Pitoňák, 2017). In addition to discrimination and exclusion, SMY are often subject to institutionalized prejudice, heterosexism, and violence. SMY are over 3.5 times as likely to be physically forced to have sex, over 2.5 times more likely to have experienced sexual dating violence, and over twice as likely to have experienced physical dating violence compared to their heterosexual peers (Centers for Disease Control and Prevention, 2017). These broad and pervasive instances of devaluation and violence often contribute to a sense of shame about one's sexual orientation and psychological and emotional difficulties (DeCamp \& Bakken, 2016; House, Van Horn, Coppeans, \& Stepleman, 2011); Meyer, 2003; Pitoňák, 2017). In fact, research demonstrates that SMY are more likely to experience depression, anxiety, feelings of isolation, lower self-esteem, and suicidal ideation compared to their heterosexual peers (Almeida, Johnson, Corliss, Molnar, \& Azrael, 2009; DeCamp \& Bakken, 2016; House et al., 2011; Pitoňák, 2017).

\subsection{NSSI behaviors of sexual minority youth}

Given the prevalence of discrimination, exclusion, violence, and psychological health disparities, it is not surprising that SMY often struggle with NSSI. As in heterosexual youth, NSSI among SMY has been consistently linked with emotional distress, including depression, anxiety, and low self-esteem (Almeida et al., 2009; DeCamp \& Bakken, 2016; House et al., 2011). In fact, numerous studies show SMY have higher rates of NSSI compared to their heterosexual peers, up to 8 times greater prevalence (Taliaferro \& Muehlenkamp, 2017). While adolescents are at an elevated risk for NSSI and suicidal ideation, SMY are especially vulnerable to both (Taliaferro \& Muehlenkamp, 2017). Thus, while SMY share many of the same risk factors for NSSI such as emotional disturbances and dysregulation, they often face unique minority stressors as a result of their stigmatized sexualities.

As presented in Fig. 1, it is proposed that SMY experience general effects (proximal and distal) along with effects that are almost exclusively a function of their sexual minority status. Past experiences of sexual minority-based discrimination, internalized oppression, and minority stress all act as distal effects that are unique to the SMY population, while homophobic bullying, social rejection, microaggressions, and institutionalized prejudice all act as proximal effects for SMY (DeCamp \& Bakken, 2016; Meyer et al., 2015; Muehlenkamp et al., 2015; Pitoňák, 2017). Furthermore, a SMY who experiences the effects of ongoing discrimination may begin to have high levels of interpersonal/intrapersonal shame (Almeida et al., 2009; DeCamp \& Bakken, 2016; House et al., 2011; Meyer, 2003). This would act as a 
distal factor and would predispose this individual to NSSI because they may perceive themselves and their sexuality negatively. Alternatively, bullying and discrimination may act as a proximal factor if the SMY encounters and copes with the emotional dysregulation caused by these stressors by NSSI (Meyer, 2003; Muehlenkamp et al., 2015). Thus, any of the distinctive social and societal stressors that SMY encounter could be a proximal and/or distal effect (depending on their effect on the individual) and contribute to an increased susceptibility to NSSI.

\section{Clinical recommendations for mental health practitioners}

\subsection{Knowledge and awareness}

Given the seriousness of NSSI and the challenges of working with SMY in a sensitive and competent manner, there are several key implications regarding clinical knowledge. First, it is important for the clinician to acquire knowledge about the history of oppression and discrimination that affects queer communities, and the resulting mental health effects previously mentioned (Moradi \& Budge, 2018). Equipped with this knowledge, clinicians can provide culturally-competent services with greater empathy, understanding, and with a stronger working alliance - therapeutic factors that many SMY do not experience in their other relationships (Almeida et al., 2009; Moradi \& Budge, 2018; Muehlenkamp et al., 2015). Second, while it is important to consider the effects of minority stress on the development of psychopathology, it is also important for therapists to understand that sexuality is just one facet of identity. Thus, it is recommended that clinicians treat the client as a whole person, rather than focusing solely on their sexual orientation as the only salient aspect of their identity (Talburt and Rasmussen, 2010). Third, having a solid understanding about the prevalence and etiology of NSSI increases the compassion clinicians can offer (Walsh, 2012).

Fourth, effective treatment depends on clinicians developing an awareness of the prejudices and biases they might hold about individuals who NSSI (Walsh, 2012). The most common biases include perceiving NSSI as a suicide attempt, a form of attention seeking, or the behavior of an irrational or dangerous person. All of these biases can stigmatize the client and minimize their pain, complicating therapeutic alliances and reducing treatment effectiveness.

Finally and most importantly, therapists should carefully monitor their reactivity to sexual minorities as they negotiate a balance between supporting and challenging their SMY client. Overly-sympathetic clinicians will be triggered by the burden of their clients' situation (proximal and distal effects) and will be hesitant to push their client to master other self-regulatory coping strategies to replace the NSSI. Alternatively, other clinicians will struggle to develop a strong working alliance with their client if their views about treating SMY populations cause them to be insufficiently empathic or too harsh when inviting changes in coping behavior. This may be especially salient for clinicians who have biases against working with sexual minorities based on clinical experiences or personal biases about normative sexuality. A careful monitoring of the therapeutic process is warranted to avoid instances of transference that may negatively impact the therapeutic alliance or treatment outcomes. This is paramount given the importance of using inclusive and non-pathologizing language about LGBQ + identities for effective and affirmative psychotherapy with SMY (Moradi \& Budge, 2018). While it is not ethical to deny a youth to care, especially on the basis of their stigmatized identity, we recommend that clinicians give their clients the option to be referred to a therapist with more expertise in working with SMY. However, it is also the therapist's responsibility to become more culturally mindful and competent when working with this population. Additional supervision may be warranted given the sensitivity of the presenting problem and the potential vulnerability of the population.

\subsection{Assessment}

The Diagnostic Statistical Manual (DSM-5) does not contain specific diagnostic criteria for NSSI, which has historically been associated with borderline personality disorder (BPD) (Sahlin, Moberg, Hirvikoski, \& Jokinen, 2015). While NSSI has commonly been linked with BPD, recent evidence suggests that NSSI is more frequently comorbid with mood disorders, such as depression and anxiety (Selby, Bender, Gordon, Nock, \& Joiner Jr, 2012). As a consequence of no official NSSI diagnosis and prevalent comorbidity, formal measures and diagnostic interviews are the most appropriate methods of diagnosing and assessing NSSI behaviors.

Among the best assessment tools is the Non-Suicidal Self-Injury Assessment Tool (NSSI-AT; Whitlock, Exner-Cortens, \& Purington, 2014), a 39-item measure used to verify NSSI and document the methods used to inflict NSSI. The NSSI-AT also assesses NSSI frequency, function, and age of onset and cessation, and can be used to explore for suicidal intent and ideation. The NSSI-AT was tested on three samples of participants who were randomly selected from eight northeast and midwest public and private universities (Whitlock, Exner-Cortens, \& Purington, 2014). This assessment has been found to have high testretest reliability (0.77) and high concurrent, convergent and discriminant validity (Whitlock et al., 2014). Another assessment is the Deliberate NSSI Inventory (Gratz, 2001), a 17-item self-report questionnaire specifically designed to detect NSSI behaviors as well as frequency, severity, duration, and type of NSSI behavior. This measure was tested on a sample of 150 undergraduate students from the University of Massachusetts Boston, who ranged in age from 18 to 64 (Gratz, 2001). Ninety-seven percent of the participants were heterosexual, and $81 \%$ of participants were single (Gratz, 2001). Similar to the NSSI-AT, the Deliberate NSSI Inventory also has high internal consistency ( $\alpha=0.82)$, high test-retest reliability, and high convergent validity.

The Inventory of Statements About Self-Injury (ISAS; Klonsky \& Glenn, 2009; Klonsky \& Olino, 2008) is another useful assessment that assesses for a variety of NSSI behaviors that have occurred intentionally and without suicidal intent. Section one of the ISAS assesses for the frequency of NSSI behaviors and the types of behaviors being utilized (e.g. burning, biting, pinching, cutting) (Klonsky \& Olino, 2008). Section two of the ISAS consists of 39 Likert-scaled questions that inquire about the function of NSSI (e.g. "When I self-harm, I am releasing emotional pressure that has built up inside of me" (Klonsky \& Glenn, 2009). This assessment demonstrates excellent internal consistency, reliability, and validity (Klonsky \& Glenn, 2009).

In addition to these written assessments, clinicians may utilize faceto-face interviews to collect additional information and confirm data collected via written assessments. The most helpful questions are aimed towards: (a) tracking the adolescent's cycle of NSSI, including the triggers and emotional states before, during, and after NSSI; (b) types of NSSI used, (c) the function of NSSI, (d) level of commitment to abstaining from NSSI behaviors, (e) length of time the client has been engaging in NSSI, and (f) other contextual information about NSSI behaviors.

\subsection{Recommended model and interventions}

Based on the existing literature on NSSI and the needs of SMY, we have synthesized tenets of DBT-A (Mehlum et al., 2014) and LGBQspecific interventions (DeCamp \& Bakken, 2016; Goldbach, 2015; Moradi \& Budge, 2018) to create a treatment model as a suggested method of treating SMY who engage in NSSI. Within this model, "A" represents distress tolerance, "B" represents emotional regulation, "C" represents mindfulness, " $D$ " represents interpersonal skills, "E" represents family therapy sessions, and " $F$ " represents intervening with minority stress. While the presentation of this model is based on existing research, it is imperative for therapists to use clinical judgement 
to determine which elements of the model would be best suited for their particular client.

Both clinical expertise and empathy are equally important when treating NSSI behaviors of SMY. The Contextual Model proposes common pathways through which psychotherapy produces benefits to clients. These pathways involve (a) the development of a therapeutic relationship and (b) agreed upon goals for clarity in treatment for optimal collaborative work and therapeutic effectiveness (Wampold, 2015). These pathways are essential to develop a bond of trust and expertise to understand the presenting problem and unique proximal and distal stressors experienced by SMY (DeCamp \& Bakken, 2016; Goldbach, 2015; Ryan, 2009; Wampold, 2015). While certainly these factors are important when intervening clinically with any population, the unique needs and experiences of SMY who engage in NSSI warrant unique treatment (Moradi \& Budge, 2018). Therefore, tailored, LGBQspecific interventions (e.g. addressing minority stress and sexual identity development) are also necessary for optimal treatment-effectiveness and to create an environment of safety and healing (Moradi \& Budge, 2018). Based on the specific needs and experiences of SMY who engage in NSSI, we recommend DBT-A treatment, treatment of minority stress, family therapy, and/or narrative interventions.

\subsubsection{DBT-A treatment for NSSI}

Nock (2010) noted that, as recently as 2010, there were no empirical treatments or prevention protocols specifically designed for NSSI. Since that time, several treatment modalities have been empirically validated for their effectiveness in reducing NSSI behaviors. In fact, in a recent meta-analysis (Ougrin, Tranah, Stahl, Moran, \& Asarnow, 2015) that reviewed randomized control trials for the treatment of NSSI, dialectical behavioral therapy (DBT; Linehan, 2015) and its adolescent specific version (DBT-A) were recommended as some of the best treatment options. Adolescents treated with DBT-A injured themselves less often, attempted suicide less often, and dropped out of therapy less often compared to conventional therapeutic models (Mehlum et al., 2014). Another study found that treatment was generally effective with a DBT-A modality, with significant reductions in the frequency of NSSI and reduced severity in both suicidal ideation and depressive symptoms (Mehlum et al., 2014).

As a clinical approach, DBT combines traditional cognitive behavioral therapy with acceptance and mindfulness approaches (Houben et al., 2017; Linehan, 2015). In fact, DBT has been so effective at treating NSSI, that it has been modified for treating adolescents by shortening treatment times, including parents and caregivers in the therapeutic process, and adding emphasis on skills deficits that are common among teenagers (Mehlum et al., 2014). DBT-A is manualized and comprehensive, while affording clinicians a flexible multimodal outpatient approach that includes weekly individual therapy and family sessions and multifamily skills training groups as needed (Fleischhaker et al., 2011; Linehan, 2015; Mehlum et al., 2014).

For a full description of DBT-A, please consult Mehlum et al., 2014 and Fleischhaker et al., 2011; however, in short, there are four primary skills that are developed in the context of DBT-A treatment: emotional regulation, distress tolerance, mindfulness, and interpersonal skills (Mehlum et al., 2014). While DBT-A is primarily behavioral and skill focused, there is a strong emphasis on mindfulness to increase one's ability to emotionally self-regulate and tolerate stressors (Houben et al., 2017). This is essential when working with NSSI in adolescents as the main factor perpetuating NSSI is one's desire to emotionally regulate.

Although no studies have been conducted that investigate DBT-A and its application to SMY who engage in NSSI, the targeted interventions are not expected to differ for this group. In accordance with Nock (2009), adolescents are influenced by distal and proximal effects, inducing intrapersonal distress which often leads to emotional dysregulation. Dysregulation is then addressed through NSSI and/or through other coping strategies (positive and negative). This dynamic process, along with the four primary DBT-A skills are presented in Fig. 1.
DBT-A focuses on four primary skills, each of which can be used to intervene at a different point in the aforementioned process of NSSI (Nock, 2009; Nock, 2010). The first skill (distress tolerance, A in Figure) increases SMY ability to withstand the impact of distal and proximal effects, thereby reducing intrapersonal distress (Linehan, 2015). The second skill (emotional regulation, B in Figure) increases adolescents' ability to cope with the intrapersonal stress (Linehan, 2015). Third, mindfulness ( $\mathrm{C}$ in Figure) acts directly as a coping mechanism when emotional dysregulation is encountered, allowing individuals to regain control over their emotions. Mindfulness also allows SMY to become more aware of their emotions and able to experience them in the present without judgement or fear (Linehan, 2015). Lastly, interpersonal skills (D in Figure) allows youth to effectively communicate their emotional dysregulation so they can reach out in a clear and direct manner for help in controlling their emotions (Linehan, 2015). In sum, DBT-A is one of the most promising empirically evidenced treatments for NSSI and may be especially helpful for SMY populations.

\subsubsection{Treating minority stress}

While NSSI behaviors can be directly targeted through models such as DBT-A, is it also imperative to intervene with minority stress ( $\mathrm{F}$ in Figure) to decrease the use of NSSI (DeCamp \& Bakken, 2016; Goldbach, 2015). During this process, it is imperative for the therapist to understand the complexity of minority stress and how both direct victimization experiences and more implicit instances of sexual-identity based victimization may contribute to NSSI. In treating SMY, it is first imperative to (a) normalize the impact of minority stress and (b) challenge maladaptive cognitions that promote the internalization of minority stress (Goldbach, 2015; Pachankis, 2015). Normalizing the use of NSSI as a coping mechanism in the face of tremendous stigma can reduce shame and decrease stress that may interfere with treatment outcomes. Therapists can help clients reflect on the messages they have received about their sexuality and how they may have internalized these into their sense of worth. Helping clients become aware of the influence of minority stress in their lives helps to move the blame from the SMY and their resilience towards a heteronormative society (Pachankis, 2015). As clinicians facilitate this self-awareness, they can assist SMY in challenging and replacing some of these internalized, stigmatized thinking with more affirmative messages.

Through this process, it is also critical to affirm and validate the client's personal strengths and healthy sexual expressions, to promote the development of self-esteem and buffer against internalized stigma. Therapists may facilitate this process by asking clients to articulate their unique strengths they possess and how they can draw upon these to facilitate resilience in the face of ongoing minority stressors. Another important intervention is to (a) facilitate emotional awareness and regulation and to (b) reduce emotional avoidance (Goldbach, 2015; Pachankis, 2015). This is crucial, given the impact of minority stress and emotional coping. Therapists should affirm queer sexualities as healthy and normative. Therapists can guide SMY through exercises to help clients consider healthy and rewarding expressions of their sexuality.

There is also considerable value in connecting SMY to other members of LGBTQ + communities. Several studies have suggested that such social integration helps to buffer against feelings of discrimination, bullying, and marginalization that often lead to the development of other mental health difficulties (Pachankis, 2015; Ryan, 2009). For stigmatized populations, connection to an affirmative community provides a safe space to receive support, socialize, and obtain a sense of belonging. (Pachankis, 2015; Ryan, 2009; Taliaferro \& Muehlenkamp, 2017). In fact, connection to important parental and nonparental support figures is protective against NSSI being used as a coping mechanism (Taliaferro \& Muehlenkamp, 2017). Given the research on the importance of social support to buffer against minority stress, clinicians should integrate interventions to strengthen interpersonal connections and empower assertive communication within interpersonal 
relationships into their treatment plan (Pachankis, 2015). This is imperative, given that SMY often face implicit messages that their needs or desires are unimportant or invalid. Thus, while facilitating the development of support, it is also crucial to empower SMY to communicate their thoughts and emotions within these relationships (Pachankis, 2015). Therapists can help combat discrimination and under-representation by supporting clients in surrounding themselves with affirmative peers, teachers, community leaders, and role models.

\subsubsection{Family treatment for NSSI among SMY}

While DBT-A can be implemented on an individual (adolescent-focused) basis, there is also considerable rationale for treating NSSI from a systemic perspective (Brent et al., 2013; Mehlum et al., 2014; Muehlenkamp et al., 2012; Ryan, 2009). Family member's reactions to their SMY's sexual orientation is either a risk or protective factor for key concerns such as sexual health, substance abuse, depression, suicidal ideation, HIV infection, identity formation, and emotional well-being (Ryan, 2009). Given that adolescents function within the context of their very influential family, family therapy has been recommended (Brent et al., 2013; Ryan, 2009) for guiding parents, siblings, and others as they negotiate the challenges of becoming lesbian, bisexual, queer, or gay-membered families. The rationale for including family therapy sessions in conjunction with DBT-A is strengthened by theories of NSSI that suggest that treatment is most effective when it focused both on promoting emotional regulation and strengthening interpersonal bonds (Muehlenkamp et al., 2012).

However, family members are often at various levels of understanding and support for their sexual minority family member. Some members, in an effort to protect their child, may try to help them fit in with heterosexual peers by limiting conversations about their child's sexual identity, trying to change their sexual orientation, or preventing them from having access to LGBQ + friends or resources (Ryan, 2009). However, these efforts are often devastating and are generally interpreted by their SMY as a form of rejection of a core aspect of their identity (Ryan, 2009). As previously mentioned, feelings of rejection and resulting emotional dysregulation may directly impact mental health and potentially, NSSI incidents.

As such, care should be taken in planning how to include family members into therapy, due to the risk that family rejection might increase the likelihood of NSSI. Thus, it is important to meet with any family members who might be disapproving before integrated family therapy takes place. Therapists should use psychoeducation about the effects of minority stress previously mentioned, showing how important it is for the SMY to receive support from their family members (Taliaferro \& Muehlenkamp, 2017). It is imperative for clinicians to coach family members on how to show love and to support their child's sexual identity, even when it feels uncomfortable or unfamiliar. Specifically, clinicians should coach family members in how to (a) express affection, (b) engage in conversations about the youth's identity, (c) advocate for the youth in various settings, and (d) require that all other family members show respect and love (Ryan, 2009). It is recommended that therapists help family members understand that they do not have to agree with the SMY's opinions or choices to be able to show warmth, love, and support. Clinicians may support family members in this endeavor while also processing through any resistance, grief, or loss that may potentially affect the expression of support.

Throughout this process, it is also critical for therapists to help all family members develop more effective communication skills to better support the SMY. A family-based treatment also allows for all family members to be exposed to better coping strategies and provides them an opportunity to custom fit the new skills to meet the unique needs of their family (Brent et al., 2013). Effective family sessions can also work to alleviate or buffer the impact of proximal/distal effects (E in Fig. 1) which should decrease the likelihood of NSSI. Further, family skills training teaches caregivers how to communicate about NSSI to negotiate better comprehensive care for the youth at school or in other settings (Brent et al., 2013).

\subsubsection{Narrative interventions for NSSI among SMY}

As another intervention to address minority stress, narrative therapy may be effective at addressing dominant discourses that have privileged heterosexuality, while labeling other types of sexuality as non-normative (McLean, 2008; Yarhouse, 2008). Narrative therapy considers how clients develop their identity and construct meaning within the context of their experiences (White \& Epston, 1990). Given the power family members have on impacting SMY's identities and psychological health, the authors theorize that each family member's narrative may intersect and affect meaning constructed around these stories (White \& Epston, 1990). Even further, the construction of these narratives may intersect with and be impacted by broader socio-cultural narratives surrounding sexuality. By examining (and challenging) the interface between one's own story and the dominant societal discourse, narrative therapy can be used to help SMY and their families find new ways to interact without as many dysfunctional or limiting communication patterns.

While the literature suggests that narrative therapy can be effective in treating minority-stress in sexual minority adults (McLean, 2008; Yarhouse, 2008), it has not yet been empirically tested with SMY who engage in NSSI. However, consistent with the adolescent's developmentally-normative vulnerability (to sociocultural forces) and engagement in identity formation, it is theorized that narrative approaches may be especially effective in treating SMY who engage in NSSI. Thus, it is suggested that therapists help the client examine past experiences, including their sexual attraction to others, with an aim on understanding how their intrapersonal distress resulted in NSSI as their elected coping mechanism. For example, as one key narrative to explore, we hypothesize that some SMY engage in NSSI behaviors as a way to punish themselves for being different compared to the heteronormative discourse in many western cultures. It is also important to explore how this narrative has been formed and reinforced (e.g. from rejecting interactions with family members and peers).

SMY who use NSSI may be stuck in negative patterns of viewing themselves, so affirmative and strength-based techniques may facilitate this process. Therapists may highlight the client's strengths and unique outcomes of being able to overcome NSSI, helping the client to develop a new lens through which to view themselves and their choices for coping mechanisms. As mentioned, internalized homophobia, discrimination, and bullying have a profound effect on the mental health of SMY. Consequently, it is hypothesized that intervening at the level of deconstructing, externalizing, and reframing how these forces have shaped their narrative may have positive implications for self-esteem, mental health, and identity. Therapists may assist SMY in determining how they would prefer to live and identify themselves by co-creating a more affirming narrative (McLean, 2008; White \& Epston, 1990; Yarhouse, 2008).

While utilizing any of these interventions with SMY, it is recommended that the main treatment outcomes be (a) promoting acceptance of one's sexual orientation (in terms of its multiple aspects of identity, attraction, and behavior) and (b) decreasing NSSI as a coping strategy. Based on the current research, it is imperative that both of these treatment goals be accomplished. Intervention may occur along various points during treatment to accomplish these goals (see Fig. 1). Ultimately, it is up to the clinician's judgement as to which goal should be the initial focus of treatment, based on the client's specific needs, the interventions being used, and the strengths of the therapist.

\subsection{Integration of theory and intervention}

Considering the previously outlined literature, using evidenced based and systemic treatments are recommended for this presenting problem and population. Grounding these treatment strategies in the theoretical framework proposed by Nock (2009) provides a more holistic integration between the suggested treatments and current theories 
used to explain NSSI. Conceptualizing NSSI as a regulating and communicative behavior that may originate from proximal and distal effects can aid therapists in more aptly targeting treatment (Muehlenkamp et al., 2012; Nock, 2009; Nock, 2010). Moreover, Nock (2009) when paired with minority stress theory may provide clinicians, SMY, and their caregivers with novel and empowering understanding of how social stigma may impact stress responses and coping behaviors (Brent et al., 2013; Goldbach, 2015; Linehan, 2015; Meyer, 2003; Nock, 2009; Nock, 2010; Ryan, 2009; Taliaferro \& Muehlenkamp, 2017). Furthermore, DBT-A is a comprehensive and flexible model that allows for the integration of family sessions (Fleischhaker et al., 2011; Linehan, 2015; Mehlum et al., 2014) and minority stress interventions to be interwoven within treatment. During the treatment process, it is suggested that narrowing DBT-A and minority stress interventions to individual components of the client's NSSI cycle may be most impactful in treatment. Fig. 1 may also provide additional visual instruction on how therapists can integrate tenets of DBT-A and systemic treatment of minority stress within Nock's framework of the etiology of NSSI.

\section{Conclusion and implications}

This article is based on a careful overview of two bodies of clinical literature: (a) treating adolescent NSSI and (b) working with SMY populations and their families.

\subsection{Social work implications}

Treating NSSI in a SMY population is a multifaceted process, as it involves treating both an understudied presenting problem and an understudied minority population. This is no small undertaking; considerable care should be given to understand the relevant literature concerning both the population and the issue of NSSI. This includes the origins of self-harm and its maintenance, the specific social forces that impact SMY. Of equal importance is the need for clinicians to develop self-awareness about personal biases that may impact treatment. Culturally competent care is imperative for treatment success and may require additional supervision.

Effective assessment is paramount for proper treatment; given there is no DSM criteria to assist clinicians, formal measures and diagnostic interviews are the best tools to aid clinicians. There are several available assessments including the NSSI-AT, the Deliberate NSSI Inventory, the ISAS, and face-to-face interviews. These enable clinicians to efficaciously to determine the scope of the NSSI and make more informed treatment decisions.

Intervention recommendations include using the empirically validated treatments for NSSI (DBT-A), as well as, using narrative family therapy to combat effects of heterosexism and heteronormativity that may influence maladaptive personal narratives. These models contain many advantageous interventions to address not just the individual's NSSI behaviors, but the family system as well. Furthermore, prompt treatment is of crucial importance for the individual and for the family, in order to reduce the effects of the emotional stress and physical damage of the NSSI as well as the risk of suicide. Expeditious treatment lessens the financial burden on both the family and the larger societal/ insurance structure.

\subsection{Future research}

While this article outlines suggested treatment approaches based on an integration of the research on treating NSSI and minority stress in SMY, the nexus between NSSI and minority stress experienced by SMY has yet to be explored and warrants future empirical investigation. Specifically, further research on family treatment approaches is warranted. Many treatment approaches have recommended the importance of family interventions, although this has received little empirical investigation. While the proposed model is based on an integration of existing literature, this area is still almost entirely unexplored, highlighting the need for future clinical research to test the acceptability and feasibility of specific interventions for this presenting problem and population. Future research is of prime importance to elucidate the develop more culturally competent and effective treatment.

\section{Conflicts of interest}

This manuscript has not been published and is not under consideration for publication elsewhere. The author has no conflicts of interest to disclose, nor funding to report.

\section{References}

Almeida, J., Johnson, R. M., Corliss, H. L., Molnar, B. E., \& Azrael, D. (2009). Emotional distress among LGBT Youth: The influence of perceived discrimination based on sexual orientation. Journal of Youth and Adolescence, 38, 1001-1014. https://doi.org/ 10.1007/s10964-009-9397-9.

Brent, D. A., McMakin, D. L., Kennard, B. D., Goldstein, T. R., Mayes, T. L., \& Douaihy, A. B. (2013). Protecting adolescents from NSSI: A critical review of intervention studies. Journal of the American Academy of Child \& Adolescent Psychiatry, 52, 1260-1271. https://doi.org/10.1016/j.jaac. 2013.09.009.

Centers for Disease Control and Prevention (2017). Youth risk behavior survey questionnaire. Available at: www.cdc.gov/yrbs.

DeCamp, W., \& Bakken, N. W. (2016). Self-injury, suicide ideation, and sexual orientation: Differences in causes and correlates among high school students. Journal of Injury \& Violence, 8, 15-24. https://doi.org/10.5249/ jivr.v8i1.545.

Fleischhaker, C., Böhme, R., Sixt, B., Brück, C., Schneider, C., \& Schulz, E. (2011). Dialectical behavioral therapy for adolescents (DBT-A): A clinical trial for patients with suicidal and self-injurious behavior and borderline symptoms with a one-year follow-up. Child and Adolescent Psychiatry and Mental Health, 5, 1-10. https://doi.org/ 10.1186/1753-2000-5-3.

Fox, K. R., Hooley, J. M., Smith, D. M., Ribeiro, J. D., Huang, X., Nock, M. K., \& Franklin, J. C. (2017). Self-injurious thoughts and behaviors may be more common and severe among people identifying as a sexual minority. Behavior Therapy. https://doi.org/10. 1016/j.beth.2017.11.009 Advance online publication.

Glenn, C. R., \& Klonsky, E. D. (2009). Social context during non-suicidal self-injury indicates suicide risk. Personality and Individual Differences, 46, 25-29. https://doi.org/ 10.1016/j.paid.2008.08.020.

Goldbach, J. T. (2015). Strategies employed by sexual minority adolescents to cope with minority stress. Psychology of Sexual Orientation and Gender Diversity, 2, 297-306. https://doi.org/10.1037/2Fsgd0000124.

Gratz, K. L. (2001). Measurement of deliberate NSSI: Preliminary data on the Deliberate NSSI Inventory. Journal of Psychopathology and Behavioral Assessment, 23, 253-263. https://doi.org/10.1023/A:1012779403943.x.

Hamza, C. A., Stewart, S. L., \& Willoughby, T. (2012). Examining the link between nonsuicidal self-injury and suicidal behavior: A review of the literature and an integrated model. Clinical Psychology Review, 32, 482-495. https://doi.org/10.1016/j. cpr.2012.05.003.

Hatzenbuehler, M. L. (2009). How does sexual minority stigma "get under the skin"? A psychological mediation framework. Psychological Bulletin, 135, 707-730. https:// doi.org/10.1037/a0016441.

Hooley, J. M., \& Franklin, J. C. (2018). Why do people hurt themselves? A new conceptual model of nonsuicidal self-injury. Clinical Psychological Science, 6, 428-451. https://doi.org/10.1177/2167702617745641.

Houben, M., Claes, L., Vansteelandt, K., Berens, A., Sleuwaegen, E., \& Kuppens, P. (2017) The emotion regulation function of nonsuicidal self-injury: A momentary assessment study in inpatients with borderline personality disorder features. Journal of Abnormal Psychology, 126, 89-95. https://doi.org/10.1037/abn0000229.

House, A. S., Horn, E., Coppeans, C., \& Stepleman, L. M. (2011). Interpersonal trauma and discriminatory events as predictors of suicidal and nonsuicidal self-injury in gay, lesbian, bisexual, and transgender persons. Traumatology, 17, 75-85. https://doi.org/ $10.1177 / 1534765610395621$.

Klonsky, E. D., \& Glenn, C. G. (2009). Assessing the functions of non-suicidal self-injury: Psychometric properties of the Inventory of statements about Self-injury (ISAS). Journal of Psychopathology and Behavioral Assessment, 31, 215-219. https://doi.org/ 10.1007/s10862-008-9107-z.

Klonsky, E. D., \& Olino, T. M. (2008). Identifying clinically distinct subgroups of selfinjurers among young adults: A latent class analysis. Journal of Consulting and Clinical Psychology, 76, 22-27. https://doi.org/10.1037/0022-006X.76.1.22.

Klonsky, E. D., Victor, S. E., \& Saffer, B. Y. (2014). Nonsuicidal self-injury: What we know, and what we need to know. Canadian Journal of Psychiatry, 59, 565-568. https://doi. org/10.1177/070674371405901101.

Lewis, S. P., \& Heath, N. L. (2015). Nonsuicidal self-injury among youth. Journal of Pediatrics, 166, 526-530.

Linehan, M. M. (2015). DBT skills training manual. New York, NY: The Guilford Press.

McLean, R. (2008). Working with gay men from a narrative counseling perspective: A case study. Journal of LGBT Issues in Counseling, 2, 243-257. https://doi.org/10. 1080/15538600802120085.

Mehlum, L., Tørmoen, A. J., Ramberg, M., Haga, E., Diep, L. M., Laberg, S., ... Grøholt, B. (2014). Dialectical behavior therapy for adolescents with repeated suicidal and self- 
harming behavior: A randomized trial. Journal of the American Academy of Child \& Adolescent Psychiatry, 53, 1082-1091. https://doi.org/10.1016/j.jaac.2014.07.003.

Meyer, I. H. (2003). Prejudice, social stress, and mental health in lesbian, gay, and bisexual populations: Conceptual issues and research evidence. Psychological Bulletin, 129, 674-697. https://doi.org/10.1037/0033-2909.129.5.674.

Meyer, I. H., Teylan, M., \& Schwartz, S. (2015). The role of help-seeking in preventing suicide attempts among lesbians, gay men, and bisexuals. Suicide and Life-threatening Behavior, 45, 25-36. https://doi.org/10.1111/sltb.12104.

Moradi, B., \& Budge, S. L. (2018). Engaging in LGBQ + affirmative psychotherapies with all clients: Defining themes and practices. Journal of Clinical Psychology, 74, 2028-2042. https://doi.org/10.1002/jclp.22687.

Muehlenkamp, J., Brausch, A., Quigley, K., \& Whitlock, J. (2012). Interpersonal features and functions of nonsuicidal self-injury. Suicide and Life-threatening Behavior, 43, 67-80. https://doi.org/10.1111/j.1943-278X.2012.00128.x.

Muehlenkamp, J. J., Hilt, L. M., Ehlinger, P. P., \& McMillan, T. (2015). Nonsuicidal selfinjury in sexual minority college students: A test of theoretical integration. Child and Adolescent Psychiatry and Mental Health, 9, 1-8. https://doi.org/10.1186/s13034015-0050-y.

Nock, M. K. (2009). Why do people hurt themselves? New insights into the nature and functions of self-injury. Current Directions in Psychological Science, 18, 78-83. https:// doi.org/10.1111/j.1467-8721.2009.01613.x.

Nock, M. K. (2010). Self-injury. Annual Review of Clinical Psychology, 6, 339-363. https:// doi.org/10.1146/annurev.clinpsy.121208.131258.

Ougrin, D., Tranah, T., Stahl, D., Moran, P., \& Asarnow, J. R. (2015). Therapeutic interventions for suicide attempts and self-harm in adolescents: Systematic review and meta-analysis. Journal of the American Academy of Child \& Adolescent Psychiatry, 54, 97-107. https://doi.org/10.1016/j.jaac.2014.10.009.

Pachankis, J. E. (2015). A transdiagnostic minority stress treatment approach for gay and bisexual men's syndemic health conditions. Archives of Sexual Behavior, 44, 1843-1860. https://doi.org/10.1007/s10508-015-0480-x.

Pitoňák, M. (2017). Mental health in non-heterosexuals: Minority stress theory and related explanation frameworks review. Mental Health \& Prevention, 5, 63-73. https:// doi.org/10.1016/j.mhp.2016.10.002.

Prinstein, M. J., Heilbron, N., Guerry, J. D., Franklin, J. C., Rancourt, D., Simon, V., \& Spirito, A. (2010). Peer influence and nonsuicidal self injury: Longitudinal results in community and clinically-referred adolescent samples. Journal of Abnormal Child Psychology, 38, 669-682. https://doi.org/10.1007/s10802-010-9423-0.

Ryan, C. (2009). Supporting families, healthy children: Helping families with lesbian, gay, bisexual, \& transgender children. San Francisco, CA: Family Acceptance Project.

Sahlin, H., Moberg, T., Hirvikoski, T., \& Jokinen, J. (2015). Non-suicidal self-injury and interpersonal violence in suicide attempters. Archives of Suicide Research, 19, 500-509. https://doi.org/10.1080/13811118.2015.1004487.

Scott, L. N., Pilkonis, P. A., Hipwell, A. E., Keenan, K., \& Stepp, S. D. (2015). Non-suicidal self-injury and suicidal ideation as predictors of suicide attempts in adolescent girls: A multi-wave prospective study. Comprehensive Psychiatry, 58, 1-10. https://doi.org/ 10.1016/j.comppsych.2014.12.011.
Selby, E. A., Bender, T. W., Gordon, K. H., Nock, M. K., \& Joiner, T. E., Jr. (2012). Nonsuicidal self-injury (NSSI) disorder: A preliminary study. Personality Disorders: Theory, Research, and Treatment, 3, 167-175. https://doi.org/10.1037/a0024405.

Sinclair, J. M., Gray, A., Rivero-Arias, O., Saunders, K. E., \& Hawton, K. (2011) Healthcare and social services resource use and costs of NSSI patients. Social Psychiatry and Psychiatric Epidemiology, 46, 263-271. https://doi.org/10.1007/ s00127-010-0183-5.

Stall, R., Friedman, M., \& Catania, J. A. (2007). Interacting epidemics and gay men's health A theory of syndemic production among urban gay men. Unequal Opportunity: Health Disparities Affecting Gay and Bisexual Men in the United States. United Kingdom: Oxford University Press251-274. https://doi.org/10.1093/acprof:oso/9780195301533.003. 0009.

Talburt, S., \& Rasmussen, M. L. (2010). 'After-queer' tendencies in queer research International Journal of Qualitative Studies in Education, 23, 1-14. https://doi.org/10. 1080/09518390903447184.

Taliaferro, L. A., \& Muehlenkamp, J. J. (2017). Nonsuicidal self-injury and suicidality among sexual minority youth: Risk factors and protective connectedness factors. Academic Pediatrics, 17, 715-722. https://doi.org/10.1016/j.acap.2016.11.002.

Umbach, T. (2013). Community health needs assessment. ManuscriptOchsner Medical Center.

Walsh, B. W. (2012). Treating self-injury: A practical guide. Guilford Press.

Wampold, B. E. (2015). How important are the common factors in psychotherapy? An update. World Psychiatry, 14, 270-277. https://doi.org/10.1002/wps.20238.

White, M., \& Epston, D. (1990). Narrative means to therapeutic ends. New York: W. W. Norton.

Whitehead, P. C., Johnson, F. G., \& Ferrence, R. (1973). Measuring the incidence of selfinjury: Some methodological and design considerations. American Journal of Orthopsychiatry, 43, 142-148. https://doi.org/10.1111/2Fj.1939-0025.1973. tb00795.x.

Whitlock, J., Exner-Cortens, D., \& Purington, A. (2014). Assessment of nonsuicidal selfinjury: Development and initial validation of the Non-Suicidal Self-Injury-Assessment Tool (NSSI-AT). Psychological Assessment, 26, 935-946. https://doi.org/10.1037/ a0036611.

Whitlock, J., \& Knox, K. L. (2007). The relationship between self-injurious behavior and suicide in a young adult population. Archives of Pediatrics \& Adolescent Medicine, 161(7), 634-640.

World Professional Association for Transgender Health (2018). Standards of care for the health of transsexual, transgender, and gender nonconforming people (7th ed.). Available at https://www.wpath.org/media/cms/Documents/SOC\%20v7/SOC\% 20V7_English.pdf.

Yarhouse, M. A. (2008). Narrative sexual identity therapy. American Journal of Family Therapy, 36, 196-210. https://doi.org/10.1080/01926180701236498.

Zetterqvist, M. (2017). Non-suicidal self-injury in adolescents: Characterization of the disorder and the issue of distress and impairment. Suicide and Life-threatening Behavior, 47, 321-335. https://doi.org/10.1111/sltb.12283. 